\title{
Design, characterization and evaluation of PEGylated- mucin for oral delivery of metformin hydrochloride
}

\author{
Momoh M. A. ${ }^{1 \star}$, Adedokun M. O. ${ }^{2}$, Adikwu M. U. ${ }^{1}$, Kenechukwu F. C. ${ }^{1}$, Ibezim E. C. ${ }^{1}$ and \\ Ugwoke E. E. ${ }^{1}$ \\ ${ }^{1}$ Drug Delivery Unit, Department of Pharmaceutics, University of Nigeria Nsukka, Enugu State. \\ ${ }^{2}$ Department of Pharmaceutical Technology and Pharmaceutical Microbiology, University of Uyo, Akwa- Ibom State.
}

Accepted 13 November, 2012

\begin{abstract}
The study was undertaken to formulate and evaluate PEGylated-mucin matrices-based solid microparticles for oral administration of metformin hydrochloride (MTH). PEGylated-mucin matrices formulated with PEG-2000 and Mucin were used to prepare metformin-loaded PEGylated-mucin using solvent interaction method. Characterizations based on size and morphology, zeta potential and polydispersity index, loading and encapsulation efficiency (EE\%) were carried out on the PEGylated matrices. In vitro release of metformin from the preparation was performed in phosphate buffer while in vivo release as a function of the antidiabetes effects were conducted in alloxan induced diabetes rats. Maximum and minimum EE\% of $\mathbf{8 1 . 0}$ and $\mathbf{4 4 . 0 \%}$ were obtained for matrices formed with PEG-Mucin ratio of $3: 1(D)$ and $0: 1(B)$, respectively. Irregular and rough matrices of size range $58.80 \pm 0.21 \mu \mathrm{m}$ to $124.1 \pm 0.1 \mu \mathrm{m}$ were produced. The release of MTH in phosphate buffer varied widely with the PEG and Mucin contents. Moreover, significant $(\mathrm{p}<0.005)$ amount of MTH was released in vivo from the matrices as demonstrated in the basal glucose reduction than the positive control. These results demonstrated that PEGylated matrices would likely to offer a reliable means of delivering metformin orally.
\end{abstract}

Key words: PEG-Mucin, diabetics, metformin, bioactivity.

\section{INTRODUCTION}

In recent time, formulation scientists have doubled their effort towards the development of novel and oral controlled release drug delivery systems to provide a long-term therapeutic concentration of drugs following a single dose. Many controlled release drug delivery systems are based on polymers and their conjugates (Rouge et al., 2006). But, there are several physiological difficulties, which include restraining/localizing the drug delivery system within the regions of the gastrointestinal tract and the high variable nature of gastric emptying process (Rouge et al., 2006). The major absorption zone, stomach or upper part of intestine can contribute to incomplete drug release from the drug delivery system leading to diminished efficacy of the administered dose.

*Corresponding author. E-mail: jointmomoh@yahoo.com. Tel: 08037784357.
Therefore, localizing the drug delivery in a specific region of the gastrointestinal tract due to its mucoadhesiveness increases the intimacy and duration of contact between the drug containing polymer and the mucous surface. Such a drug delivery system offers numerous advantages, especially for drugs exhibiting an absorption window or for drugs with a stability problem in the stomach. Overall, the intimate and prolonged contact of the drug delivery system with the absorbing membrane has the potential to maximize the rate of drug absorption (Chowdary and Srinivasa, 2003; Pothal et al., 2004). These considerations have lead to the development of oral controlled release microcapsules /microspheres possessing mucoadhessive properties (Pothal et al., 2004).

Metformin is an oral anti-hyperglycemic agent, which shows incomplete absorption from the gastrointestinal tract and the absolute bioavailability of 50 to $60 \%$ with relatively short plasma half-life of 1.5 to $4.5 \mathrm{~h}$. Oral absorption of metformin is confined to the upper part of 
the intestine (Indian Pharmacopoeia, 1996). Therefore, the low bioavailability can be ascribed to a comparatively high pre-systemic clearance that takes place after administration. More importantly, are the gastrointestinal side effects such as abdominal discomfort, nausea, and diarrhea associated with metformin intake and the need for repeated administration to maintain an effective plasma concentration, that at times decreases patient compliance (Murugesh, 2003). It may therefore be more desirable to deliver the drug in a sustained release dosage form. However, contributions by pharmaceutical and biomaterials scientists have led to the development of novel carriers to be used as innovative drug-delivery systems. The performance of these systems was improved by synthesizing polymers that have desirable chemical, physical and biological properties, and favourable interactions with biological materials. These advancements have started to unlock ways around the problems that are encountered with oral drug delivery (Davis, 2006).

Among these measures, PEGylation is one of the most popular methods. PEG (polyethylene glycol) is a nontoxic and non-irritant hydrophilic polymer (Zabaleta et al., 2007). PEGylation of proteins, drugs, liposomes, and nanoparticles has been proven to be an effective approach for extending circulation in the blood stream, owing to the steric hindrance of the PEG chains (Gao et al., 2007; Hong et al., 2004). Similarly, PEGylated polymers may improve the stability of the drug delivery system in the blood by preventing protein absorption and uptake by reticuloendothelial systems (RES) (Tsutsumi et al., 1999; Shibata et al., 2004). Mucins are high molecular weight glycosylated proteins, believed to be the major structure-forming component of mucus (Bloofield, 1983) and responsible for the cohesive and visco-elastic nature of mucus gel (Mortazavi et al., 1992). Pure mucin is the glycoprotein part of the mucus devoid of water, free proteins, minerals and lipids (Adikwu et al., 2005). Apart from acting as protectants and lubricants, mucins are known to be the substrate on which mucoadhesive polymers attach, thus the interest in hybridizing them with other polymers (Builders, 2008).

PEGylation-based polymers have been extensively used for mucoadhesive applications because they exhibit high adhesive bond strengths on contact with tissues (Lowman et al., 1997). This increases residence time and drug bioavailability in most cases. Researchers believe that, in such systems, the free PEG chains that were grafted onto the PEGylation act as mucoadhesive anchors, causing an increase in mucoadhesion and making these carriers very promising as systems for drug delivery (Lowman et al., 1997). Scientists race to discover better ways to improve the adhesive properties of polymers such as using linear PEG as an adhesion promoter, neutralizing ionic polymers and developing polymer combinations to provide better drug delivery system (Momoh et al., 2010; Nakamura et al., 2004).

The primary objective of this study was to develop PEGylated-mucin based microparticulate delivery system that would enhance the sustained release of metformin hydrochloride which will lead to improved bioavailability of the drug.

\section{MATERIALS AND METHODS}

The following materials were used: polyethylene glycol 2000 (Cary Roth, Germany), monobasic potassium phosphate, sodium hydroxide and concentrated hydrochloric acid (BDH, England), distilled water (Lion water, University of Nigeria Nsukka). Metformin hydrochloride pure sample was obtained as a gift from Farmex Meyer Pharma Ltd. (Ikeja, Lagos State, Nigeria). Other reagents were of analytical grade and used without further purification.

\section{Extraction of snail mucin}

After procurement, shells of the giant African land snails were knocked open at the apex and a spirally coiled rod inserted to remove the fleshy body from where the excretory parts were removed. The fleshy parts were then placed in $250 \mathrm{ml}$ of water and washed several times until the slimy mucin was completely washed off. These washings were pooled together in an aluminium bucket, precipitated with chilled acetone and then dried by lyophilization. Briefly, the mucin mucilage was rapidly frozen in an ethanolic bath at $-25^{\circ} \mathrm{C}$ for $25 \mathrm{~min}$ and then placed in a vacuum chamber of the lyophilizer (Labconco Freezone apparatus, USA). The lyophilisation was carried out at $-25^{\circ} \mathrm{C}$ under vacuum (1.20 to $3.0 \mathrm{mbar}$ ) for $24 \mathrm{~h}$. The greyish-brown lyophilized flakes of the snail mucin were collected and pulverized into fine powder using a mortar and pestle and stored in an airtight container until used.

\section{Preparation of unloaded PEGylated-mucin}

Unloaded PEGylated-mucin matrices were prepared by solvent method. Briefly, $5 \mathrm{~g}$ of PEG and Mucin were dissolved separately in $30 \mathrm{ml}$ of highly purified water with a magnetic stirring set up (300 $\mathrm{rpm}$ ) in a $100 \mathrm{ml}$ beaker until a clear solution was obtained. After 4 $\mathrm{h}$, the mucin mix was then dispersed in the PEG solution using magnetic stirring (300 rpm) and allowed to stand undisturbed for 24 h. A $500 \mathrm{ml}$ volume of chilled acetone maintained at $-20^{\circ} \mathrm{C}$ was then slowly added into $250 \mathrm{ml}$ beaker containing the pegylate with constant stirring at a speed of $100 \mathrm{rpm}$, for $30 \mathrm{~min}$, in an ice to regulate the temperature (owing to the nature of mucin). The PEGylated-mucin samples formed were collected by filtration through a milliport filter $0.22 \mu \mathrm{m}$. Detail of the ratios are shown in Table 1.

\section{Loading of metformin into the pegylated-matrices}

Metformin was loaded into the various matrices by the diffusion loading method. A 2.0 g-quantity of the pegylated-mucin matrices (Table 1) was placed in a $50 \mathrm{ml}$ beaker and $500 \mathrm{mg}$ quantities of the metformin previously dissolved in $5 \mathrm{ml}$ of highly purified water, added. This was allowed to stand for $3 \mathrm{~h}$ in an ice jar. The resulting matrices after hydration were freeze dried and then sieved using a standard sieve of size $100 \mu \mathrm{m}$. The matrix was stored in an air tight container and maintained at $10^{\circ} \mathrm{C}$ until used for further study. 
Table 1. Ratios of mucin and PEG used for preparing unloaded microparticles.

\begin{tabular}{ccc}
\hline Batch code & PEG & Mucin \\
\hline A & 1 & 1 \\
B & 0 & 1 \\
C & 1 & 2 \\
D & 3 & 1 \\
E & 1 & 3 \\
\hline
\end{tabular}

\section{Characterisation of unloaded PEGylated-mucin matrices}

\section{Morphology, particle size and zeta potential analysis}

The morphology of the PEGylated-mucin matrix was obtained by field emission scanning electron microscopy (SEM) (JEOL JSM$6500 \mathrm{~F}$, Tokyo, Japan) under an accelerated voltage of $4 \mathrm{KV}$ and a working distance of $6 \mathrm{~mm}$. A drop of sample dispersion was spread onto a metal slab and the excess droplets removed with a filter paper. The samples were then coated in a cathode evaporator with a fine carbon layer and observed by scanning electron microscope (SEM). The particle size of the PEGylated-mucin matrix was determined by photon correlation spectroscopy using a Zetasizer nano (ZEN 3600, Malvern, UK). Size distribution analysis was performed according to an earlier report (Nakamura et al., 2004). Briefly, the samples were diluted with highly purified water and the electrophoretic mobility determined at $25^{\circ} \mathrm{C}$ and a light-scattering detection angle of $90^{\circ} \mathrm{C}$. The electrophoretic mobility values obtained were used to calculate the zeta potentials employing a computer software (DTS Version 4.1 Malvern, UK).

The mean particle size and polydispersity index were determined. The zeta potential was similarly determined using a Zetasizer by phase analysis light scattering.

\section{Differential scanning calorimetry (DSC)}

The degree of crystallinity and changes in heat capacity of the PEGylated-mucin was determined using a calorimeter (DSC $220^{\circ} \mathrm{C}$ ) connected to a disc station (Mettler Toledo, Greifensee, Switzerland). Briefly, about 4 to $5 \mathrm{mg}$ of the PEGylated-mucin was weighed into an aluminium pan, sealed hermetically, and the thermal behavior determined in the range of 20 to $220^{\circ} \mathrm{C}$ at a heating rate of $5^{\circ} \mathrm{C} / \mathrm{min}$ and then cooled back to $20^{\circ} \mathrm{C}$. This analysis was carried out in all the samples of the PEGylated-mucin. The baselines were determined using an empty pan, and all the thermograms were baseline-corrected. Transition temperatures were determined from the endothermic peak minima while transition enthalpies (where possible) were obtained by integration of the endothermic transitions. The results were expressed as the mean of three independent measurements.

\section{Determination of drug loading (DL) and encapsulation efficiency (EE)}

About $50 \mathrm{mg}$ of metformin-loaded microparticles were dissolved in $10 \mathrm{ml}$ of phosphate buffer ( $\mathrm{pH} \mathrm{7.4)}$ and added into a microconcentrator (5000 MWCO Viva science, Germany). This was centrifuged (TDL-4 B. Bran Scientific and Instru. Co. England) at $1500 \mathrm{rpm}$ for $120 \mathrm{~min}$. The supernatants were adequately analyzed with a spectrophotometer (Jenway 60172, Germany) at $242 \mathrm{~nm}$. The amount of drug encapsulated in the microparticles was calculated reference to a standard Beer's plot to obtain the \% encapsulation efficiency (EE) using the formula:

$$
\mathrm{DL}(\mathrm{EE})=\frac{\text { Total quantity of the drug- quantity in supernatant }}{\text { Total quantity of the carrier }} \times 100
$$

\section{In vitro drug release}

The in vitro release profiles of the metformin-loaded microparticles were determined. Approximately, a $100 \mathrm{mg}$ quantity of the metformin-loaded microparticles was filled into hard gelatin capsules. Each capsule was then placed in a $250 \mathrm{ml}$ beaker containing $200 \mathrm{ml}$ of phosphate buffer solution PBS (pH 7.4). Agitation of the fluid system $(100 \mathrm{rpm})$ was done with a magnetic stirrer. At determined intervals, $1 \mathrm{ml}$ samples were withdrawn and fresh PBS added. The withdrawn samples were filtered through a $0.22 \mu \mathrm{m}$ Millipore ${ }^{\circledR}$ filter and analyzed for metformin content using a spectrophotometer (Shimadzu, A160, Japan) at $242 \mathrm{~nm}$.

\section{Effect of the preparation on basal blood glucose level}

\section{Experimental rats}

Wistar strain albino rats of both sexes weighing between 160 to 200 $\mathrm{g}$ were bred in the Department of Biochemistry, University of Nigeria, Nsukka. The animals were housed in standard environmental conditions and kept at body temperature of $37^{\circ} \mathrm{C}$ using warming lamps. All the animals were fasted for $12 \mathrm{~h}$, but were allowed free access to water, before commencement of the experiments.

\section{Induction of experimental diabetes}

Rats of either sex weighing 160 to $200 \mathrm{~g}$ were fasted for $18 \mathrm{~h}$ before the induction of diabetes. Diabetes was induced with single intraperitoneal injection of $0.5 \mathrm{ml}$ alloxan monohydrate dissolved in normal saline $(0.9 \% \mathrm{NaCl})$ at a dose of $200 \mathrm{mg} / \mathrm{kg}$ body weight. Alloxan is known to inactivate the pancreatic cells without reducing exocrine functions. Blood glucose levels were monitored daily for 5 to 7 days using a Glucometer (Accu-Check, Switzerland) for all the rats until the induction and stabilization of diabetic state. After a week of the alloxan treatment, rats with frequent urination, loss of weight, and blood glucose levels higher than $200 \mathrm{mg} / \mathrm{dl}$ were selected and randomly divided into six groups. Before the administration of testing agent, animals were fasted overnight with free access to water. Animal ethical procedures were strictly followed in accordance with the requirements of the Ethical Committee, Faculty of Pharmaceutical Sciences, University of Nigeria, Nsukka.

\section{Administration of metformin loaded-PEGylated-mucin matrices}

The 24 diabetic rats were randomly divided into six groups of four with each group housed in a separate cage. The different formulations of the metformin-loaded microparticles were filled into hard gelatin capsules, with each capsule containing microparticles equivalent to metformin dose of $10 \mathrm{mg} / \mathrm{kg}$ body weight for each animal. The capsules were administered orally to the animals according to their weight as follows: rats in group one received formulation labeled as batch $A$, the second group received formulation 
$D$, rats in group three received formulation $E$, while groups four, five and six received marketed sample (M-S), metformin $\mathrm{HCl}$ dispersed in distilled (D-W) and distilled water (W), respectively. In order to evaluate the effect of formulations on glycemia, groups four and five were used as positive control and group six served as negative control. In each case, the formulation was administered using gastric nasal tube. Blood samples were taken from the tail of the rats at predetermined time intervals of up to $24 \mathrm{~h}$ after drug administration and examined for the basal blood glucose level using a glucometer (Accu-Check, Switzerland).

The post-dose levels of the blood glucose were expressed as a percentage of the pre-dose level. The percent basal blood glucose concentration was plotted against time for the various groups.

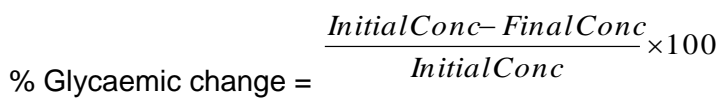

\section{Data analysis}

All experiments were performed in replicates $(n=3)$ for validity of statistical analysis. Results were expressed as mean \pm SD. ANOVA and Student's t-tests were performed on the data sets generated using Origin for Windows. Differences were considered significant for $p$ values $<0.05$.

\section{RESULTS AND DISCUSSION}

\section{Morphology, particle size and zeta potential}

Preliminary thermal analysis carried out on PEGylatedmucin matrices shows that there was an interaction between the mucin and PEG-2000 (Momoh, 2010). Hence, this method was employed to pegylate mucin and PEG-2000 which was used in this work. The SEM photomicrographs of the unloaded PEGylated-mucin matrices of batch 1 to 5 obtained by scanning electron microscopy are shown in Figures $1 \mathrm{a}$ to e. The micrographs (batch $A$ to $C$ ) show the formation of non spherical microparticles with rough porous surfaces and irregular shape. Batches $D$ and $E$, though similar in shape to batches $A$ to $C$ are characterized by larger and less spherical particles with denser and rougher surfaces. This was further confirmed by particle size analysis, which showed that size distributions vary from 72.0 to $124.0 \mu \mathrm{m}$. There was a clear distribution of both small and large particles with little agglomeration. Study has shown that the morphology of polymers used in drug delivery plays an important role in controlling the release characteristics of formulated drugs (Jain et al., 1998). This is because the size and number of pores determine the rate and extent of drug release from the polymer matrices. Generally, the shape and size of the particles are affected by the combination ratios of PEG and mucin. The mean particle size range of the unloaded PEGylated- mucin matrix as determined by photon correlation spectroscopy Zetasizer nano was found to be 71.0 to 124 $\mu \mathrm{m}$ (Table 2). There were significant variations in their particles sizes and the narrowest and densest range of particle dispersion are found in preparation $C$ and $D$, respectively. The PDI values of the various preparations $A$ to $E$ were $0.745,0.885,1.000,1.000$ and 1.000 , respectively. Generally, PDI is a measure of the distribution of molecular mass in a given polymer sample.

Values in the range of 0.95 to 1.00 are considered to be the best for particles intended for oral drug delivery (Jain et al., 1998). Values are usually greater than 1, but as the polymer chains approach uniform chain length, the value becomes 1.000 which is considered as the best as seen in preparations $\mathrm{C}$ to $\mathrm{E}$, and is mucin concentration dependent, although preparation $D$ proved otherwise. The size distribution patterns of the preparations show a unimodal distribution. On the other hand, zeta potential values varied between -18.7 to $-23.2 \mathrm{mV}$ as shown in Table 2. The significance of zeta potential is that its value can be related to the stability of colloidal dispersions (Bell, 2004). For molecules and particles that are small enough, a high zeta potential will confer stability, because the solution or dispersion will resist aggregation (Bell and Rodriguez, 2004). When the potential is low, attraction exceeds repulsion and the dispersion will break and flocculate. Those with high zeta potential (negative or positive) are, therefore, electrically stabilized while colloids with low values tend to coagulate or flocculate (Hackley et al., 2002). Zeta potential is widely used for quantification of the magnitude of the electrical charge at the double layer. Although, it is not equal to the stern potential or electric surface potential in the double layer, it is often the only available path for characterization of double-layer properties and can be calculated using theoretical models and an experimentally-determined data.

When the results of electrophorectic mobility were compared, PEG: mucin (0:1) matrices B and PEG: mucin (3:1) D had lower mobility values of -1.479 and -1.521 , respectively as compared to those of PEG: mucin (1:1) of matrices A, PEG: mucin (1:2) C and PEG: mucin (1:3) E, which were $-1.870,-1.810$ and $-1.823 \mathrm{mS} / \mathrm{cm}$, respectively (Table 2). The zeta potentials and electrophoretic mobilities determined were mucin concentration-dependent. The magnitude of zeta potential gives an indication of the potential stability of a dispersion system. Large negative or large positive zeta potential is required for dispersion stability. The general dividing line between stable and unstable suspension is generally taken as either +30 or $-30 \mathrm{mV}$ (Kosmulski and Rosenholm, 2004). The relatively high negative zeta potentials of the produced mucin PEGylation would contribute to stability of the dispersion. From the data, it is evident that all the formulations are stable in solid form 
Table 2. Characterization of the PEGylated- mucin matrices.

\begin{tabular}{cccccccccc}
\hline Batch & PEG & Mucin & Z-ave & PDI & ZP & Mob & LD & $\%$ EE & Particle size \\
\hline A & 1 & 1 & 412 & 0.745 & -23.0 & -1.870 & $34 \pm 0.4$ & $49 \pm 0.2$ & $71.1 \pm 0.41$ \\
B & 0 & 1 & 1283 & 0.885 & -18.7 & -1.479 & $23 \pm 0.1$ & $44 \pm 0.1$ & $114.2 \pm 1.4$ \\
C & 1 & 0 & 4487 & 1.000 & -23.1 & -1.810 & $30 \pm 0.2$ & $63 \pm 0.4$ & $58.8 \pm 0.21$ \\
D & 3 & 1 & 569 & 1.000 & -19.4 & -1.521 & $56 \pm 0.6$ & $81 \pm 0.0$ & $124.1 \pm 0.1$ \\
E & 1 & 3 & 2863 & 1.000 & -23.2 & -1.823 & $52 \pm 0.1$ & $74 \pm 0.3$ & $76.6 \pm 0.11$ \\
\hline
\end{tabular}

$\mathrm{PDI}=$ Polydispersibility index, $\mathrm{ZP}=$ zeta potential, $\mathrm{Mob}=$ mobility, $\mathrm{Z}$-ave $=$ average particle diameter, LD $=$ Loading dose, EE $=$ encapsulation efficiency.

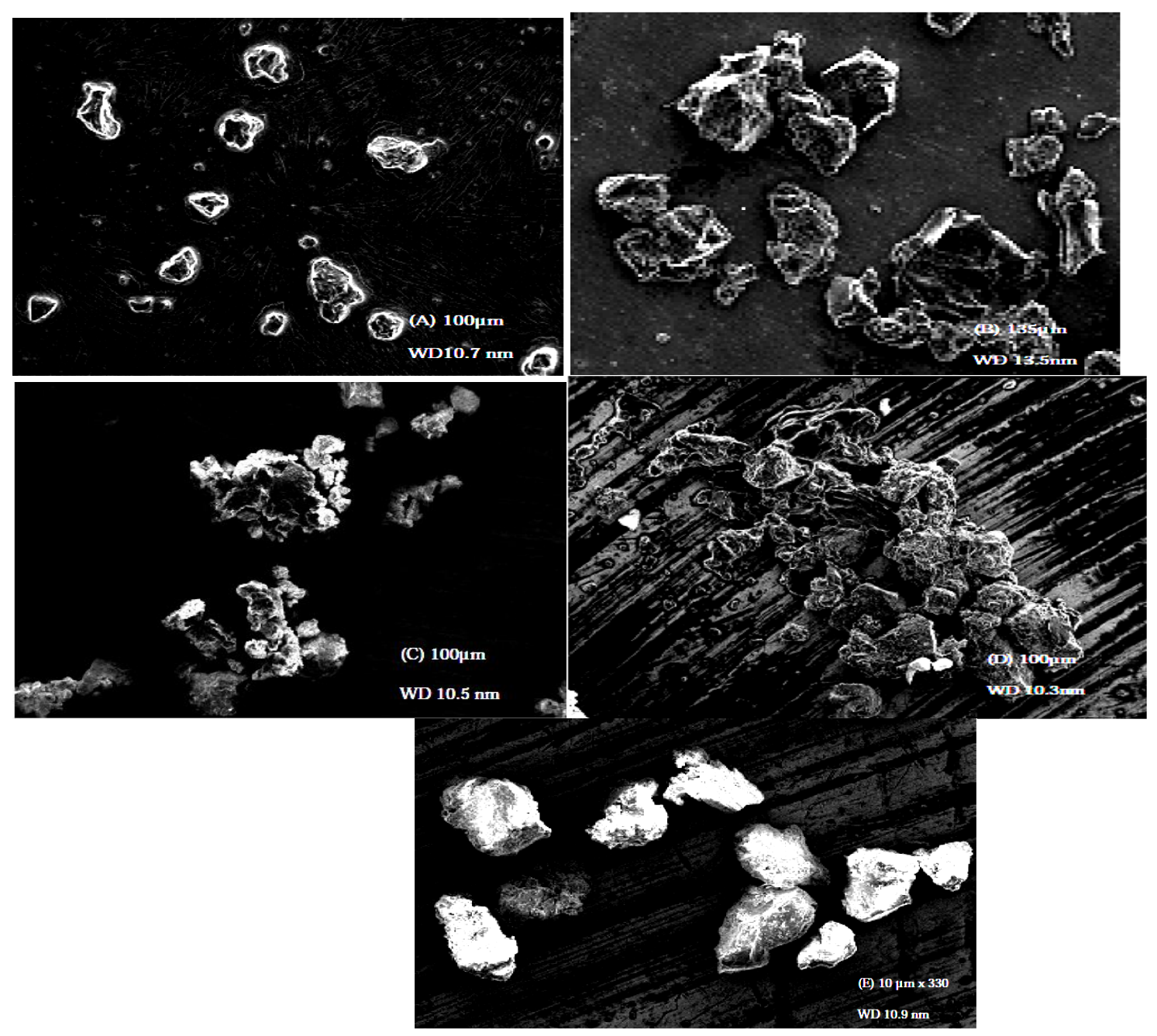

Figure 1. Scanning electron microscopy of unloaded PEGylated-mucin matrices (A-E).

but very unstable in a colloidal state.

\section{The DSC study on the PEGylated samples}

The melting behaviours and consequently, the likely release potentials of the PEGylated products were investigated by DSC. Low enthalpy transitions were obtained for some of the preparations as presented in Figures $2 \mathrm{a}$ to e. The thermotropic phase behaviour of a non-ionic amphiphilic polymer PEG is highly affected by the presence of mucin, and the related thermodynamic 
(A)

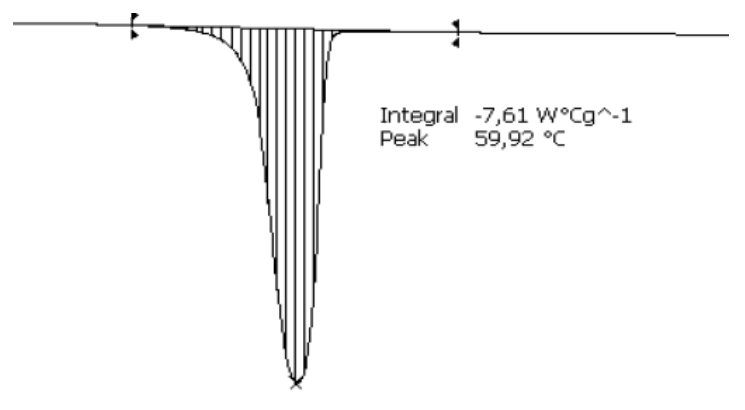

(B)

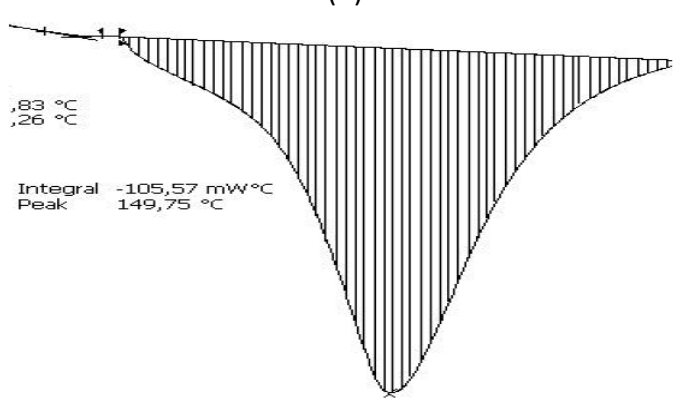

\begin{tabular}{cccccccccc}
1 & 1 & 1 & 1 & 1 & 1 & 1 & 1 & 1 & 1 \\
40 & 50 & 60 & 70 & 80 & 90 & 100 \\
\hline
\end{tabular}
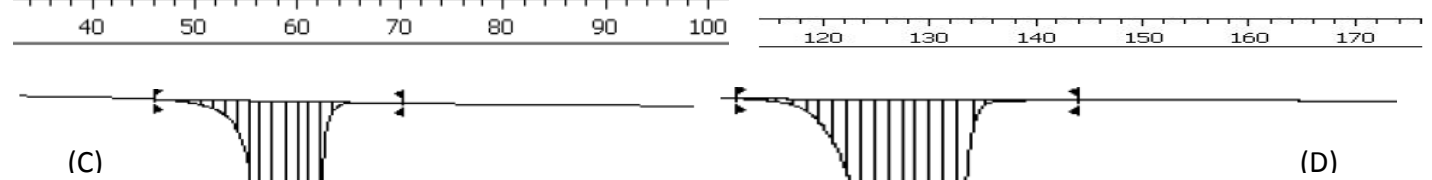

(C)
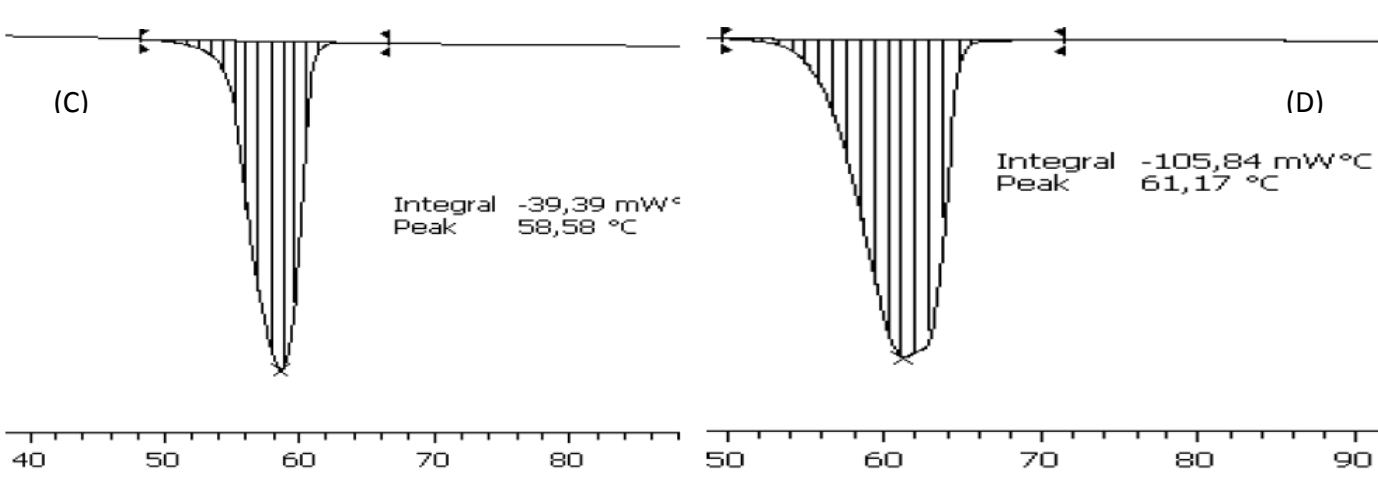

60

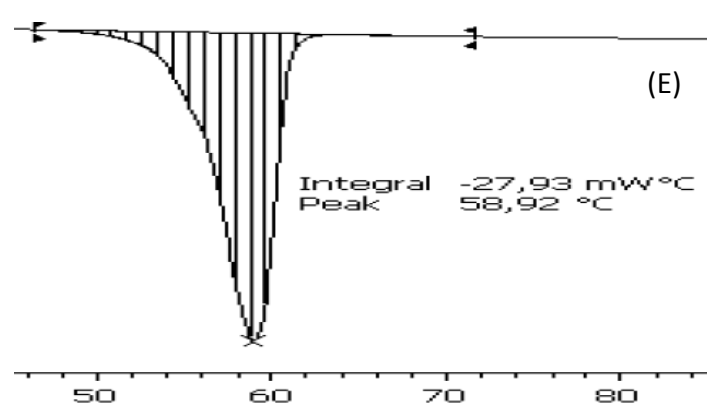

Figure 2. DSC thermographs of the unloaded PEGylated-mucin of (1:1) (a), (0:1) (b), (1:0) (c), (3:1) (d) and $(1: 3)(e)$.

variables (melting temperature and enthalpy changes) depend on the nature of the interaction between the two components (mucin and PEG). A very broad peak and two shallow endothermic peaks were detected for the pure mucin prepared without PEG with an enthalpy at low temperature of $70^{\circ} \mathrm{C}$ and the two enthalpy peaks appeared very shallow at about 250 and $325^{\circ} \mathrm{C}$ (Figures $2 a$ to e). Batches A, C, D and E showing close peaks at a low enthalpy of $70^{\circ} \mathrm{C}$, with two additional transition peaks at 350 and $425^{\circ} \mathrm{C}$ of PEG prepared with mucin were obtained for each formulation $A$ and $C$, respectively. The high enthalpy suggests high crystallinity and possible release of the drug that may be entrapped into the preparation when used to deliver drug over time.

The compressed results of the thermograms obtained for preparation containing PEG and mucin were drastically reduced, while some peaks were absent, indicating the interaction between the two components produced a single component.

\section{Encapsulation and loading efficiency}

Table 2 shows the metformin encapsulation loading efficiency of the PEGylated-mucin matrices formulated with the various ratios of PEG and mucin. The formulation was freeze dried to minimize loss of mucin integrity due to heat which will eventually affect the drug availability. High drug loadings were observed in Batches $D$ to $E$. Metformin loading generally increased with 


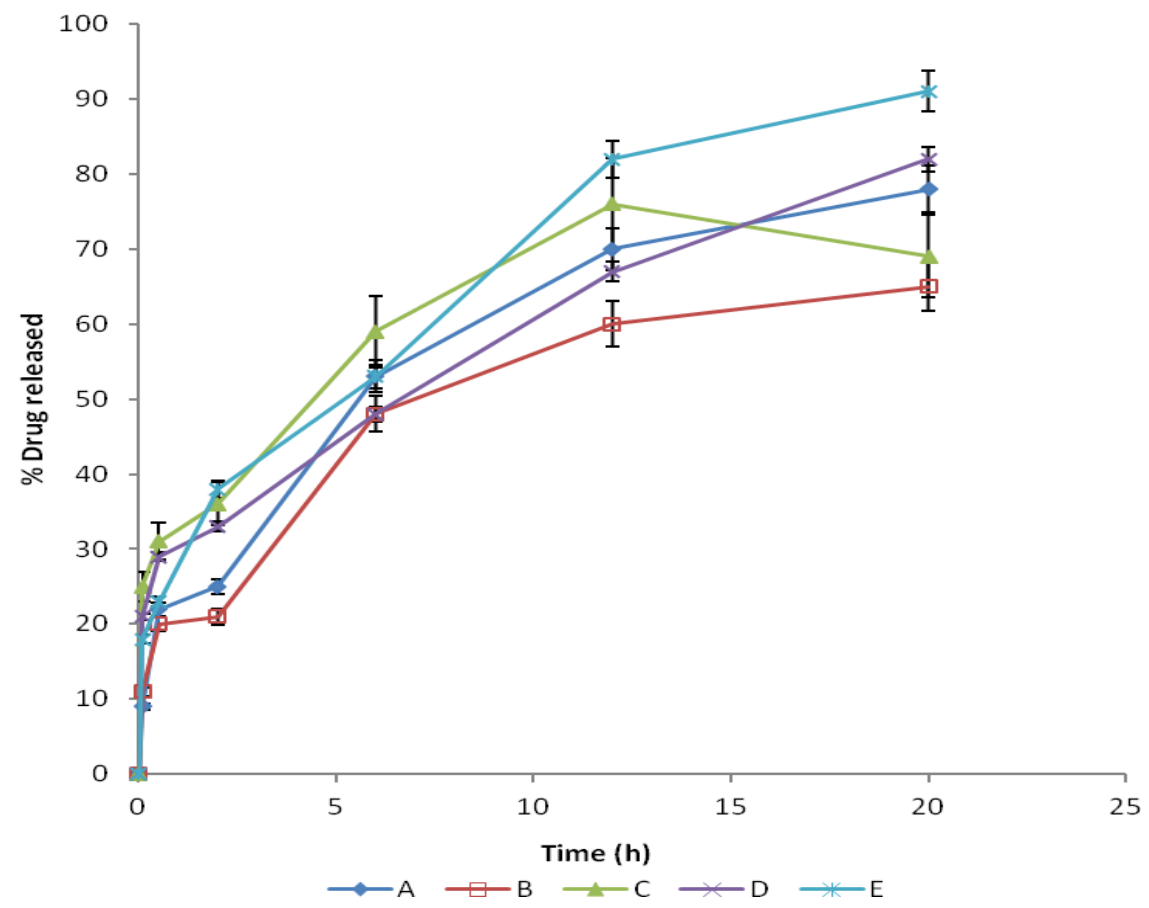

Figure 3. In vitro release profile of metformin from PEGylated-mucin matrix $[A=(1: 1)$ of PEG-Mucin, $B=(0: 1)$ of PEG-Mucin, $C=(1:)$ of PEG-Mucin, $D=(3: 1)$ of PEG-Mucin and $E=(1: 3)$ of PEG-Mucin]. Each of the batches contained $500 \mathrm{mg}$ of metformin $\mathrm{HCl}$.

of the drug was released from the capsule within the first $1.5 \mathrm{~h}$, followed by a mass release within $20 \mathrm{~h}$ the study lasted. However, the initial release (burst effect) may be an indication of the unencapsulated metformin which possibly only adsorbed to the surface of the PEGylated material, hence, the ease of the drug to get into the release medium at a fast rate. The second release observed here could be attributed to slow migration of the drug that get into the core of the PEGylated-mucin to the surface and finally into the medium. However, the release rates of metformin were prolonged in the case of preparations $C$ and $D$ than observed in $B$ and $C$, the loaded non-PEGylated polymers (PEG or Mucin), although there was no significant difference in the release rate of drug from these preparations.

Metformin is a known hydrophilic drug and belongs to the class III compounds according to the BCS. Therefore, the release rate of metformin from the PEGylated-mucin matrix systems was dependent on the mucin more than the PEG used.

\section{Result of glucose lowering effect}

The hyperglycaemic state of the rats after the administration of alloxan was monitored for $24 \mathrm{~h}$. This was to ensure that there was a persistent raise in blood glucose level before the commencement of the study. Other signs such as polyurea, polyphagia and polydypsia (3Ps) were obviously present. Alloxan became the first diabetogenic chemical agent when Dunn and Letchie accidentally produced islet-cell necrosis in rabbits while researching the nephrotoxicity of uric acid derivatives. Alloxan is a specific toxin that inactivates the pancreatic $\beta$ cells, provoking a state of primary deficiency of insulin without affecting other islet types (Dunn et al., 1943). Hence, alloxan was selected to induce diabetes in the present study. Based on the release study result, batch $A, D$ and $E$ were selected for in vivo evaluation on animal model. Results in Figure 4 indicate a good hypoglycemic effect with significant glycemia decrease for all the formulations. The mean blood glucose baseline (initial glucose level) value was taken as the $100 \%$ level and all other blood glucose level/time data were calculated as a percentage of the baseline. Rats in group (W) that received distilled water maintained a very high blood glucose level. No apparent hypoglycaemic response was observed in this group that served as negative control.

Orally administered metformin dispersed in distilled (labelled DW) water resulted in a slight fall in the blood glucose level within $0.5 \mathrm{~h}$ of administration, and maintaining the temperature up to $6 \mathrm{~h}$. The percentage blood glucose reduction for the formulations administered was significantly $(p<0.05)$ higher than the marketed 


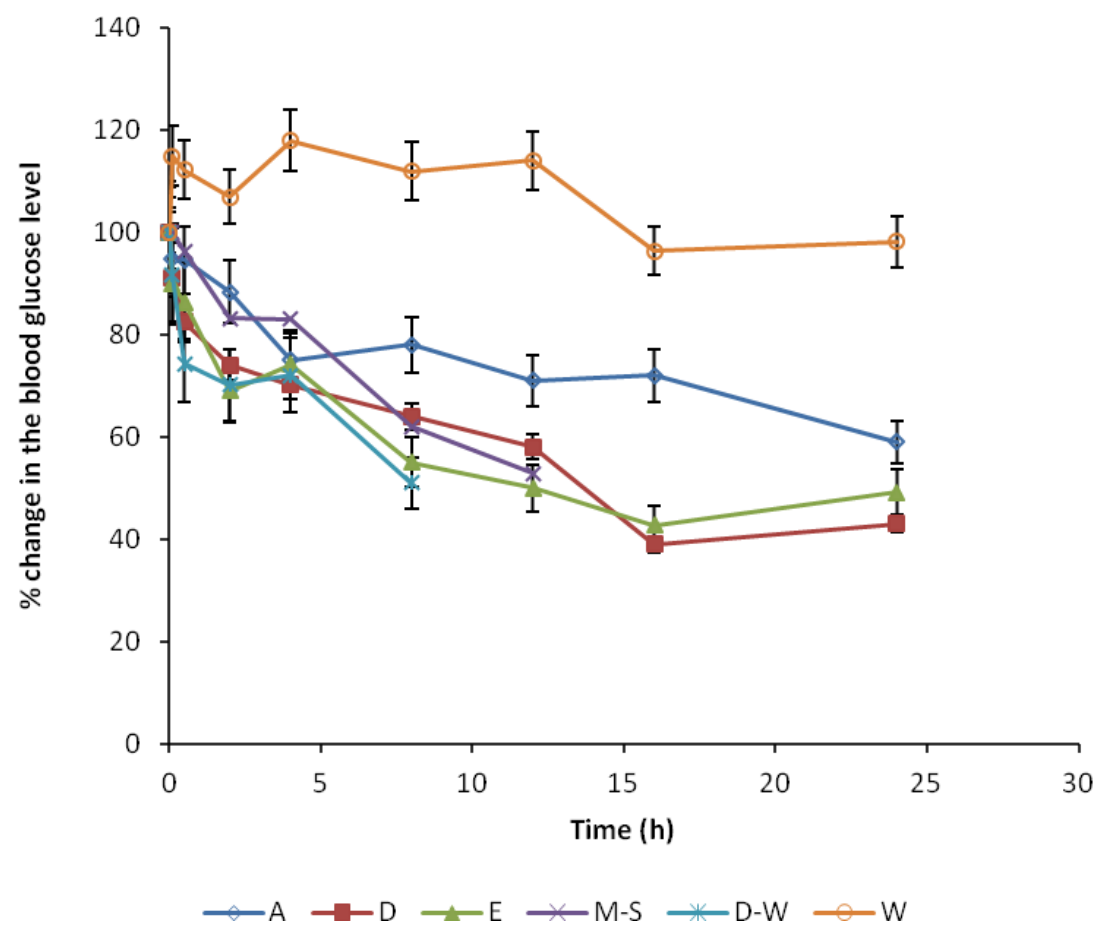

Figure 4. Effect of metformin loaded PEGylated-mucin on hyperglycaemic rats $[A=(1: 1)$ of PEG-Mucin, $D=(3: 1)$ of PEG-Mucin and $E=(1: 3)$ of PEG-Mucin, M-S $=$ Marketed sample, $\mathrm{D}-\mathrm{W}=$ Metformin dispersed in distilled water and $\mathrm{W}=$ distilled water]. Each of the batches (A, D and E) contained $500 \mathrm{mg}$ of metformin $\mathrm{HCl}$.

sample (M-S) and the dispersed pure metformin powder (D-W). All the metformin-loaded PEGylated-mucin matrix ( $A, D$ and $E$ ) prepared with the various ratio of mucin and PEG generally reduced blood glucose lowering effect higher than those of either marketed sample or and the dispersed metformin powder in distilled water. The high blood glucose reduction resulting from the formulations as compared to the positive controls indicates that there was effective delivery of the drug to the site where absorption takes place in the GIT. The formulation (batch E) produced high blood glucose reduction between 14 to $16 \mathrm{~h}$ after oral administration that was more than the marketed drug, and the effect lasted up to $24 \mathrm{~h}$, while that of positive controls only lasted for $8 \mathrm{~h}$. Batch $\mathrm{D}$ which contain PEG and mucin in the ratio of 3:1 show a similar effect to batch $E$, but with a slight reduction in the amount of glycemic effect (Figure 4). Batch A of the formulation PEG and mucin (1:1) significantly lowered the blood glucose level at the initial stage and declined within a short period of time.

Mucin being a viscoelasticity material would create a better adherence to the small intestine would lead to increased residence time, release at the delivery site, and would presumably affect the bioavailability of the drug. The mean turnover time of the mucous gel layer has been shown to vary between 47 and $270 \mathrm{~min}$, which would indicate a significant factor in designing mucoadhesive drug-delivery systems (Des Rieux et al., 2006). The mucous gel layer itself represents an unstirred water layer which impedes drug, diffusion and adsorption across the epithelium. Consequently, a prolonged release effect as observed in all the formulations, but more in batch $\mathrm{E}$ with high concentration of mucin. However, the hydrophilicity nature of PEG may have contributed to the observed effect on the basal blood glucose reduction as seen in formulation $D$ that contained high concentration of PEG ratio to mucin (3:1) in the in vivo study.

Recent studies have shown that factor such as hydrophilicity and mucoadhesion are capable of influencing drug release from the formulations and increase the transport of this system through the mucosa surfaces (Kenneth et al., 2010). In addition, the PEG chains are able to diffuse through the mucus and enhance mucoadhesion thereby enhance the release of the drug at the absorption site easier and faster than mucin (Kenneth et al., 2010).

\section{Conclusion}

PEGylated-mucin used in the study showed good 
characterization based on the parameters measured. The in vitro model of the present study has proved to be reliable to predict release of metformin HCL through the uses of PEGylated-mucin matrix which was confirmed in the in vivo glucose lowering study. This method of drug delivery may be a promising technique in the area of drug discovery and development. PEGylated-mucin loaded metformin showed slightly higher release and maintained longer times when compared with immediate-release marketed products.

The glucose lowering effect was more in the formulations ( $C$ and $D)$ than the commercially marketed metformin. This formulation could potentially reduce dosing frequency to once daily compared with two or three times daily for immediate-release formulations.

\section{ACKNOWLEDGEMENTS}

This work is a part of the thesis submitted for the award of Ph.D. by the Department of Pharmaceutics, University of Nigeria, Nsukka, Nigeria. The authors thank Farmex Meyer Pharma Ltd, Ikeja, Lagos State for the gift of metformin hydrochloride. Dr. Philip Builders of NIPRD is highly appreciated for his contribution. Dr. M. A. Momoh is grateful to the Islamic Education Trust Fund (IETF) and Nigeria-Saotome Principe for their financial support.

\section{REFERENCES}

Adikwu MU, Aneke KO, Builders PF (2005). Biophysical properties of mucin and its use as a mucoadhesive agent in drug delivery: current development and future concepts, Nig. J. Pharm. Res. 4: 60-69.

Bloofield VA (1983). Hydrodynamic properties of mucoglycoproteins, Biopolym. 21:214-221.

Bell N, Cesarano J, Voight JA, Lockwood SJ, Dimos DB (2004). Colloidal processing of chemically prepared zinc oxide varistors. Part 1. Milling and dispersion of powder. J. Mat. Res. 19(5):1333-1340.

Bell N, Rodriguez MA (2004).Dispersion properties of an alumina nanopowder using molecular, polyelectrolyte, and steric stabilization. J. Nanosci. Nanotechnol. 4: 283-290.

Builders PF, Kunle OO, Adikwu MU (2008). Preparation and characterization of mucinated agarose: a mucin-agarose physical crosslink, Int. J. Pharm. 356: 174-180.

Chowdary KPR, Srinivasa RY (2003). Preparation and Evaluation of Mucoadhesive Microcapsules of Indomethacin. Ind. J. Pharm. Sci. 65(1):49-52.

Davis S (2006). Insulin, Oral Hypoglycemic Agents, and the Pharmacology of the Endocrine Pancreas, Brunton LL, Lazo JS, Parker PK (Eds), p. 1641. McGraw-Hill, Columbus, $\mathrm{OH}$.

Des Rieux A, Fievez V, Garinot M, Schneider YJ, Préat (2006). Nanoparticles as potential oral delivery systems of proteins and vaccines: a mechanistic approach. J. Cont. Rel. 116:1-27.

Dunn JS, Sheehan HL, Mclechie NG (1943). Necrosis of langerhans produced experimentally. Lancet. 241:484-487.
Gao JQ, Eto Y, Yoshioka Y, Sekiguchi F, Kurachi S, Morishige T, Yao XL, Watanabe H, Okada H, Mukai Y, Tsutsumi Y, Mayumi T, Okada $\mathrm{N}$, Nakagawa $S$ (2007). Effective tumor targeted gene transfer using PEGylated adenovirus vector via systemic administration. J. Cont. Rel. 122:102-110.

Hackley V, Patton JI, Lum L, Wasche RJ, Naito M, Abe H, Hotta H, Pendse $H$ (2002). Analysis of the isoelectric point in moderately concentrated alumina suspensions using electroacoustic and streaming potential methods. J. Disp. Sci. Tech. 23:601-617

Hong JW, Park JH, Huh KM, Chung H, Kwon IC, Jeong SY (2004). PEGylated polyethy- lenimine for in vivo local gene delivery based on lipiodolized emulsion system, J. Cont. Rel. 99:167-176.

Indian Pharmacopoeia (1996). Government of India Ministry of Health and Social Welfare. The Controller of Publications, Delhi, 1:469-470.

Jain R, Shah NN, Malick AW (1998). Controlled drug delivery by biodegradable poly(ester) devices: different preparative approaches. Drug Dev. Ind. Pharm.14:703-727.

Kenneth O, Gerhard W, Gert F, Conrad C (2010). Matrix-loaded biodegradable gelatin nanoparticles as new approach to improve drug loading and delivery. Eur. J. Pharm. And BioPharm. 76:1-9

Kosmulski M, Rosenholm JB (2004). High ionic strength electrokinetics, Adv. Colloid Interfac. Sci. 112:93-107.

Lowman AM, Dziubla AD, Peppas NA (1997). "Novel Networks and Gels Containing Increased Amounts of Grafted and Crosslinked Poly(Ethylene Glycol)". Polym. Prepr. 38(1):622-623.

Momoh MA, Adikwu MU, Ibezim EC (2010). Formulation and evaluation the bioadhesive properties of drug delivery system based on PEGylated mucin matrices, Asian Pacif. J. Trop. Med. (2010):412415

Mortazavi SA, Carpenter BG, Smart JD (1992). Comparative study on the role played by mucus glycoprotein in the rheological behaviors of the mucoadhesive/mucosal interaction, Int. J. Pharm. 94:195-201.

Murugesh N (2003). "Oral antidiabetic drugs" In: A concise textbook of pharmacology, $5^{\text {th }}$ Edition, Sathya Publishers, Madurai, 3:196-197.

Nakamura K, Murray RJ, Joseph JI, Peppas NA, Morishita M, Lowman AM (2004). Oral insulin delivery using $P(M A A-g-E G)$ hydrogels: effects of network morphology on insulin delivery characteristics, J. Cont. Rel. 95:589- 599.

Nicholas A, Peppas J, Brock T, James M (2009). Molecular Aspects of Mucoadhesive Carrier Development for Drug Delivery and Improved Absorption. J. Biomat. Sci. 20:1-20

Pothal RK, Sahoo SK, Chatterjee S, Sahoo D, Barik BB (2004). Preparation and Evaluation of Mucoadhesive Microcapsules of Theophylline. The Ind. Pharmaci. 3(28):74-79.

Rouge N, Buri P, Doelker E (1996). Drug absorption sites in the gastrointestinal tract and dosage forms for site-specific delivery. Int. J. Pharm. 136:117-39.

Shibata H, Yoshioka Y, Ikemizu S, Kobayashi K, Yamamoto Y, Mukai Y, Okamoto T (2004). Functionalization of tumor necrosis factor-alpha using phage display technique and PEGylation improves its antitumor therapeutic window, Clin. Cancer Res. 10:8293-8300.

Tsutsumi Y, Tsunoda S, Kaneda Y, Kamada H, Kihira T, Nakagawa S, Yamamoto Y, Horisawa Y, Mayumi T (1996). In vivo anti-tumor efficacy of polyethylene glycol-modified tumor necrosis factor-alpha against tumor necrosis factor-resistant tumors. J. Cancer Res. 87:1078-1085.

Zabaleta V, Campanero MA, Irache JM (2007). An HPLC with evaporative light scattering detection method for the quantification of PEGs and Gantrez in PEGylated nanoparticles, J. Pharm. Biomed. 44:1072-1078. 